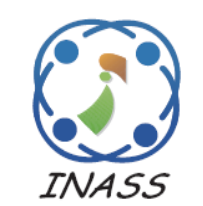

\title{
Identifying Dominant Characteristics of Students' Cognitive Domain on Clustering-based Classification
}

\author{
Yuni Yamasari ${ }^{1,2 *} \quad$ Supeno M. S. Nugroho ${ }^{1,3} \quad$ Kayo Yoshimoto $^{4}$ \\ Hideya Takahashi $^{4} \quad$ Mauridhi H. Purnomo ${ }^{1,3}$ \\ ${ }^{1}$ Department of Electrical Engineering, Institut Teknologi Sepuluh Nopember, Surabaya, Indonesia \\ ${ }^{2}$ Department of Informatics, Universitas Negeri Surabaya, Surabaya, Indonesia \\ ${ }^{3}$ Department of Computer Engineering, Institut Teknologi Sepuluh Nopember, Surabaya, Indonesia \\ ${ }^{4}$ Department of Electrical and Information Engineering, Osaka City University, Sumiyoshi-ku, Osaka, Japan \\ * Corresponding author’s Email: yuniyamasari@unesa.ac.id; hery@ee.its.ac.id
}

\begin{abstract}
The rapid growth of information and communications technology-based educational tools generates a large volume of student data with many features (characteristics). However, the mining process in the clustering task of student data is not often done optimally, so the performance of the system decreases. To overcome this problem, we propose a discretization method on logistic regression to determine the most optimal number of clusters. Additionally, we introduce a technique that combines the features selection using a filter- and wrapper-based procedures (HFS) to identify the dominant features of the students' cognitive domains. Furthermore, we evaluate the identification result by three clustering methods, namely: K-means, EM, and Farthest first. Finally, we propose the clustering-based classification so the results can be measured by using the classification metrics. Here, we apply two evaluation techniques, namely: cross-validation and percentage split. The experimental results indicate that our approach describes predominance, in terms of classification metrics over conventional methods. Our approach is around 10,847-11,134 percent higher in terms of accuracy average than the original features on both the assessment techniques. Also, this approach significantly reduces the time taken to create a prototype between 0.0167-0.027 seconds. This gives the impact on a significant reduction in the model created to the number of unsuitable students on classes based on the cognitive domain, namely: 3-12 students.
\end{abstract}

Keywords: Classification, Clustering, Student, Features selection, Cognitive domain.

\section{Introduction}

Recently, almost all processes in education rely on information and communications technology to make it better concerning the quality [1]. This dependency generates a large volume of educational data and triggers research in Educational Data Mining (EDM). EDM itself is defined as a new data mining grounds-based discipline, for example, method, task, and algorithm. It is to investigate those data to discover a descriptive pattern and predictions that characterize behaviors, assessments, educational functionalities, achievements, domain knowledge content and applications of learners [2].
Besides, EDM is done using statistical, data mining and machine learning algorithms [3].

One of the educational data is student data consisting of features that describe the characteristics of students [4-6]. The most popular task in educational data mining for those student data is classification. The previous research does in mining various student data for the prediction, such as psychomotor [7], academic performance [8, 9], and the learning result [10]. Other research classifies the data to predict the early failure of the student's academic on a specific subject [11] and to predict a persuasive message to change the student's attitude [12]. Then, the classification is carried out to analyze the student's behavior on online learning 
Table 1. The previous works in the student clustering

\begin{tabular}{|c|c|c|c|c|}
\hline Authors & $\begin{array}{l}\text { Feature } \\
\text { Discretization }\end{array}$ & $\begin{array}{l}\text { Feature } \\
\text { Selection/ } \\
\text { extraction }\end{array}$ & $\begin{array}{l}\text { The evaluation } \\
\text { technique }\end{array}$ & Description \\
\hline $\begin{array}{l}\text { Cerezo et } \\
\text { al.[4] }\end{array}$ & No & No & The statistical analysis & $\begin{array}{l}\text { Clustering of students' interaction patterns } \\
\text { on LMS using EM and K-means }\end{array}$ \\
\hline $\begin{array}{l}\text { Singh et } \\
\text { al.[17] }\end{array}$ & No & No & The silhouette score & $\begin{array}{l}\text { Clustering of students' academic } \\
\text { performance using K-means }\end{array}$ \\
\hline $\begin{array}{l}\text { Najdi et } \\
\text { al.[18] }\end{array}$ & No & No & $\begin{array}{l}\text { The clustering result } \\
\text { focuses on the analysis of } \\
\text { cluster members }\end{array}$ & $\begin{array}{l}\text { Clustering of students' typologies using K- } \\
\text { means }\end{array}$ \\
\hline Li et al.[19] & No & No & $\begin{array}{l}\text { The clustering process is } \\
\text { a step in a system }\end{array}$ & $\begin{array}{l}\text { Clustering of student academic } \\
\text { performance using Fuzzy-C-Means (FCM) } \\
\text { for a predictive model }\end{array}$ \\
\hline Fan et al.[20] & No & No & $\begin{array}{l}\text { The accuracy level and } \\
\text { the clustering time. No } \\
\text { discussion of this level. }\end{array}$ & $\begin{array}{l}\text { Clustering of college student using K- } \\
\text { means algorithm improved by the value of } \\
\text { the density threshold to remove outliers }\end{array}$ \\
\hline $\begin{array}{l}\text { Yamasari et } \\
\text { al.[21] }\end{array}$ & No & $\begin{array}{l}\text { the } \\
\text { category- } \\
\text { based } \\
\text { feature } \\
\text { extraction }\end{array}$ & $\begin{array}{l}\text { The accuracy level } \\
\text { around } 69 \%\end{array}$ & $\begin{array}{l}\text { Clustering of students' achievement using } \\
\text { Fuzzy-C-Means. The research focus to } \\
\text { enhance the clustering performance using } \\
\text { the feature extraction based on category }\end{array}$ \\
\hline $\begin{array}{l}\text { Yamasari et } \\
\text { al.[23] }\end{array}$ & No & $\begin{array}{l}\text { Yes, the } \\
\text { random } \\
\text { selection }\end{array}$ & The silhouette index & $\begin{array}{l}\text { Clustering of students' psychomotor } \\
\text { domain using K-means. The initial of } \\
\text { cluster center point using K-means++ to } \\
\text { improve the cluster validity. }\end{array}$ \\
\hline $\begin{array}{l}\text { Shankar et } \\
\text { al.[31] }\end{array}$ & No & No & The silhouette index & $\begin{array}{l}\text { Clustering of students' attributes with } \\
\text { respect to their country for analyzing of } \\
\text { their performance with K-means }\end{array}$ \\
\hline $\begin{array}{l}\text { Rosa et } \\
\text { al.[33] }\end{array}$ & No & No & $\begin{array}{l}\text { Statistical analysis: } \\
\text { - Univariate Anova } \\
\text { - Spearman correlation } \\
\text { - Crosstabulation }\end{array}$ & $\begin{array}{l}\text { The clustering of national and school } \\
\text { examinations-based high schools using } \\
\text { FCM }\end{array}$ \\
\hline $\begin{array}{l}\text { Campagni et } \\
\text { al.[34] }\end{array}$ & No & No & Pearson's correlation & $\begin{array}{l}\text { The students' clustering for recognizing } \\
\text { strategies to improve the scheduling of } \\
\text { exams and the performance of students } \\
\text { using K-means }\end{array}$ \\
\hline $\begin{array}{l}\text { Harwati et } \\
\text { al.[35] }\end{array}$ & No & No & $\begin{array}{l}\text { The outcomes of the } \\
\text { clustering depend on the } \\
\text { group members ' study }\end{array}$ & $\begin{array}{l}\text { The mapping of students' performance } \\
\text { using K-means }\end{array}$ \\
\hline $\begin{array}{l}\text { Park et } \\
\text { al.[36] }\end{array}$ & No & No & $\begin{array}{l}\text { The clustering result } \\
\text { depends on the group } \\
\text { members ' study }\end{array}$ & $\begin{array}{l}\text { The clustering of students' online behavior } \\
\text { using Latent Class Analysis (LCA) }\end{array}$ \\
\hline
\end{tabular}

[13] and to study the performance of undergraduate students [14]. However, in the previous work, almost all research still considers the whole features or characteristics in the mining process. Meanwhile, data sets often contain irrelevant features causing the performance of the system to decrease or not optimal [15]. Moreover, the data processing consumes expensive computational time [16] because this process involves all features.

Another favorite task in EDM is clustering. The previous research does the clustering task for analyzing the student's performance [17], for identifying student's typologies [18] and for predicting the student's academic performance [19]. In other research, the clustering task is applied to modeling the student career [20]. Overall, the previous studies implement the clustering method whose cluster number is determined by the user. This is a crucial step because an incorrect number of clusters drop the performance of the process.

To improve the performance of the system, methods are explored, for example, feature extraction, features selection, etc. In the research [21, 22] researchers do the extraction of features relating to student's achievements. Meanwhile, the other research applies features selection on student's psychomotor domain [23], on student's academic performance [24, 25]. Here, the previous studies have not yet made a combination of feature selection 
methods to take advantage of these methods so that the mining process has not been optimized.

In the context of a students' cognitive domain, the low performance of the system generates invalid information which leads the decision-maker to take the wrong action. For example, a student is mapped to an incorrect cluster (a good student is clustered as a poor student). As a result, the student is treated incorrectly. Moreover, accurate information is critical for a teacher to determine the appropriate teaching method to enhance the capability of the students.

Nevertheless, some previous research is not much which focuses on the discretization method and feature selection to improve the performance level of student clustering. Though, in terms of accuracy, the performance still lows namely: 69\% measured by supervised evaluation. Furthermore, almost all of the evaluation of the clustering result is done by the unsupervised evaluation for examples: silhouette index, analysis statistics, etc. (see Table 1).

Therefore, in this paper, we intend to work on those problems by enhancing the performance of the mining process. For this purpose, we extend the previous work [26] to determine an optimal number of clusters. Then, we propose a hybrid method that combines feature selection methods, namely: a filter-based and a wrapper-based approach, to eliminate irrelevant features and to improve the accuracy level. Also, we apply three clustering methods: K-means, Expectation Maximum and Farthest first. It is predicted that relevant features are the dominant characteristics of a student based on the cognitive domain. Furthermore, we propose clustering-based classification such that the cluster evaluation can be measured with the performance metrics of the classifier. Finally, the dominant characteristics identified to support a teacher to map the students' cognitive domain simpler and faster. It is because the mining process only considers a few features.

The remaining of this paper is managed in the following way. Section 2 describes the related work. Section 3 presents the proposed methods. Section 4 represents the results of the experiment and the comparison of the clustering methods. Finally, the conclusion is provided in the last section.

\section{Related work}

In this section, we discuss related work about feature selection and the clustering evaluation.

\subsection{Feature selection}

There are two categories of feature selection methods, namely: wrapper-based and filter-based approaches [27]. Wrapper-based subset selection (WBSE) is built by a classifier to estimate the worth of each feature subset. Alternatively, Filter-based subset evaluation (FBSE) was submitted to solve the issue of the redundant feature [28]. FBSE evaluates the whole subset with a multivariate way, eliminates irrelevant features and explores the relationship degree between them. FBSE is the method based on a heuristic that exploits statistical measures and probabilities to look for and evaluate the utility of all features that have been known.

Furthermore, there is another method called WBSE. It usually has better predictive accuracy than FBSE [29]. This is because each feature subset is evaluated by a particular classification algorithm. So, a feature selection approach is more optimal. However, this WBSE needs the most time to evaluate each set of features by a classification algorithm causing WBSE to be the more expensive to be executed. Moreover, wrappers are more difficult to shift from one classifier to another since the selection process requires re-initiation. Additionally, they are associated with the classifier's algorithms. Dissimilar with wrappers, FBSE explores distance measures and correlation functions to select criteria of filters [15].

Relating to feature selection in the education area, many researchers explore this method for better performance of tasks in data mining. Deepika et al. [30] propose feature selection method blending Relief-F and Budget Tree-Random Forest to improve the performance of student academic Performance Prediction. W. Punlumjeak et al. [27] explore feature selection methods consisting of FBSE (information gain, genetic algorithms, and minimum redundancy and maximum relevanceMRMR) and WBSE (SVM). To evaluate these methods, they apply four classifiers: naïve bays, decision tree, k-nearest neighbor $(\mathrm{k}-\mathrm{NN})$ and neural network. The highest performance is achieved by combination MRMR and k-NN. Then, research [24] propose FBSE method consisting of CfsSubsetEval, ChiSquaredAttributeEval and is evaluated by 14 classifiers. After, they have compared each other. It is found that the combination of random forest and principal components is the best accuracy. These researches [28] also employ FBSE method for selecting features, namely: Chi-Square, to improve the analysis sentiment on teaching evaluation. The 


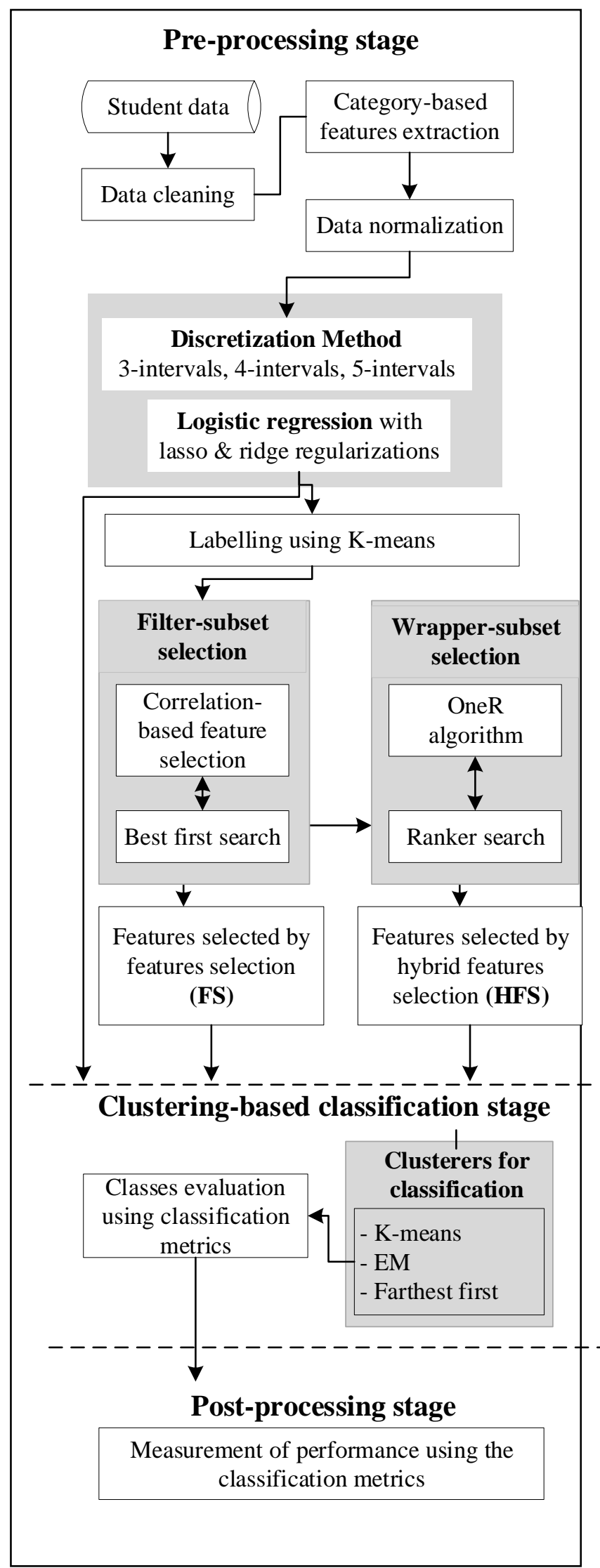

Figure. 1 The proposed framework experimental result indicates that the combination of the Chi-Square and vote ensemble method is significantly efficient.

While, the research in [29] apply Best-first search method as the wrapper, and gain information as the filter. After these methods are evaluated with many classifiers, the wrapper method shows the best method for all classifiers in improving the accuracy of student's academic performance. In an educational area, researchers often apply Filterbased subset evaluation (FBSE) because the method is addressed to solve the issue of the redundant feature. This problem must be avoided in the context of the modeling of students' achievement.

\subsection{Clustering evaluation in educational data mining}

Evaluation of data mining is intended to measure the result of the mining process. Generally, there are two evaluations of data mining, namely: supervised evaluation and unsupervised evaluation [15]. The difference between both is whether the evaluation process involves an external index or not. Supervised evaluation requires external indices, for example, labels. Accordingly, the clustering evaluation mostly is categorized as the unsupervised evaluation because this task only works the evaluation by the internal indices. Relating to the evaluation clustering, the previous research in [31] apply the clustering task by K-means to analysis the performance of student learning and measure the clustering performance using unsupervised evaluation, namely: silhouette index.

The other research also evaluates its performance by silhouette [23]. Next, using the silhouette index as unsupervised evaluation also is applied by research [17]. On the other side, the research [20] evaluates the clustering result using supervised evaluation, namely: accuracy and time.

Relating to student clustering, the preprocessing phase of some previous research seldom applies the feature selection. Moreover, the hybrid of feature selection method is not yet exploited in the students' cognitive domain for enhancing the clustering performance.

Furthermore, the majority of previous works apply the unsupervised evaluation to measure the student model in the clustering task. However, in the students' cognitive domain, the interpretation of clusters as classes is needed to generate information relating to the misclassified student. Therefore, the clustering-based classification needs to be exploited. 


\section{Methodology}

In this research, our dominant characteristics identification approach consists of three stages: preprocessing, clustering-based classification and postprocessing. In detail, we explain our proposing about the discretization method and feature selection method in the pre-processing stage and clusteringbased classification as meta-algorithm in the mining process stage. Fig. 1 illustrates the proposed framework in identifying the dominant characteristics of students.

\subsection{Pre-processing stage}

In this section, before we employ the discretization method and features selection, we do many steps, namely: data cleaning, category-based features extraction, and data normalization.

In the research, the first step is data cleaning to produce student data not containing the duplicate record, etc. [16]. Here, a record is eliminated because it contains null variables. Then, we do category-based feature extraction as our previous work to improve clustering performance [21]. In data normalization step, features are normalized to achieve the normal distribution by formula as follows:

$$
x^{\prime}=\frac{x-\mu}{\sigma}
$$

Where $\mathrm{x}, \mu$ and $\sigma$ are respectively original feature vector, mean of the feature vector and standard deviation. In the next step, we propose the discretization method on logistic regression before the feature selection is done. This step is very important because we apply the cluster algorithms that the determination of the cluster number is set by the user, namely: K-means, EM, and Farthest-first.

This method is carried out to know the fit number of clusters to generate an optimal model. Here, we adopt an equal width-based discretization method to optimize this process. Equal width is categorized as an unsupervised binning method to transform numerical variables into categorical. This algorithm splits the data into $\mathrm{k}$ intervals having an equal size. The formula of intervals width and interval boundaries are as follows:

$$
\begin{aligned}
& W=\left(V_{\max }-V_{\min }\right) / k \\
& V_{\text {min }}+W, V_{\text {min }}+2 W, \ldots, V_{\text {min }}+(k-1) W
\end{aligned}
$$

Where, $\mathrm{W}, \mathrm{V}_{\min }$ and $\mathrm{V}_{\max }$ are the interval width, the maximum and minimum values respectively. Furthermore, we propose to apply this method to three-interval, four-interval, and five-interval. We decide at these intervals because the previous research generally divides student data from three to five groups. For examples: three groups [32], [33], [34], [35], [5], four groups [36], [20], [4], and five groups.

We combine the discretization method and logistic regression to find the best interval with two regularizations, namely: lasso and ridge. Additionally, we set the evaluation technique with the percentage split. The evaluation result of all intervals is measured by the correlation value and average accuracy level on all splits. The discretization result is very important to decide the number of groups in the labeling step. Here, we apply the K-means to generate label our student data. This step also supports to evaluate the clustering using the classification metrics.

Then, we also propose feature selection methods. In this step, we propose the combination of both the filter and wrapper approaches to enhance the accuracy level. In the first step, we build a filterbased approach called FS (Features Selection). Here, we generate a new set $\mathrm{N}$ of reduced features by doing the process on the original features $\mathrm{M}$, where $\mathrm{N} \subseteq \mathrm{M}$.

We propose the Correlation Feature Selection (CFS) having robustness in deleting irrelevant and redundant features. CFS can do it because of the relationship between features evaluated by Eq. (4).

This formula defines the function of merit, which is used to pick a subset $\mathrm{S}$ consisting of $\mathrm{k}$ number of characteristics. Determining both unnecessary and redundant characteristics is achieved by $\overline{r c f}$ providing that element's relationship means to its unit. In the meanwhile, $\overline{r f f}$ is the relationship mean among features.

$$
M S=\frac{k \overline{r c f}}{\sqrt{k+k(k-1) \overline{r f f}}}
$$

CFS is a filter-based algorithm evaluating subsets of features based on a heuristic evaluation function with the hypothesis "A good feature subset is one that contains features highly correlated with the class, yet uncorrelated with each other" [37]. So, we apply a heuristic search, namely: Best first as a search function. Best first use greedy hill-climbing augmented with a backtracking facility to search the space of feature subsets. 


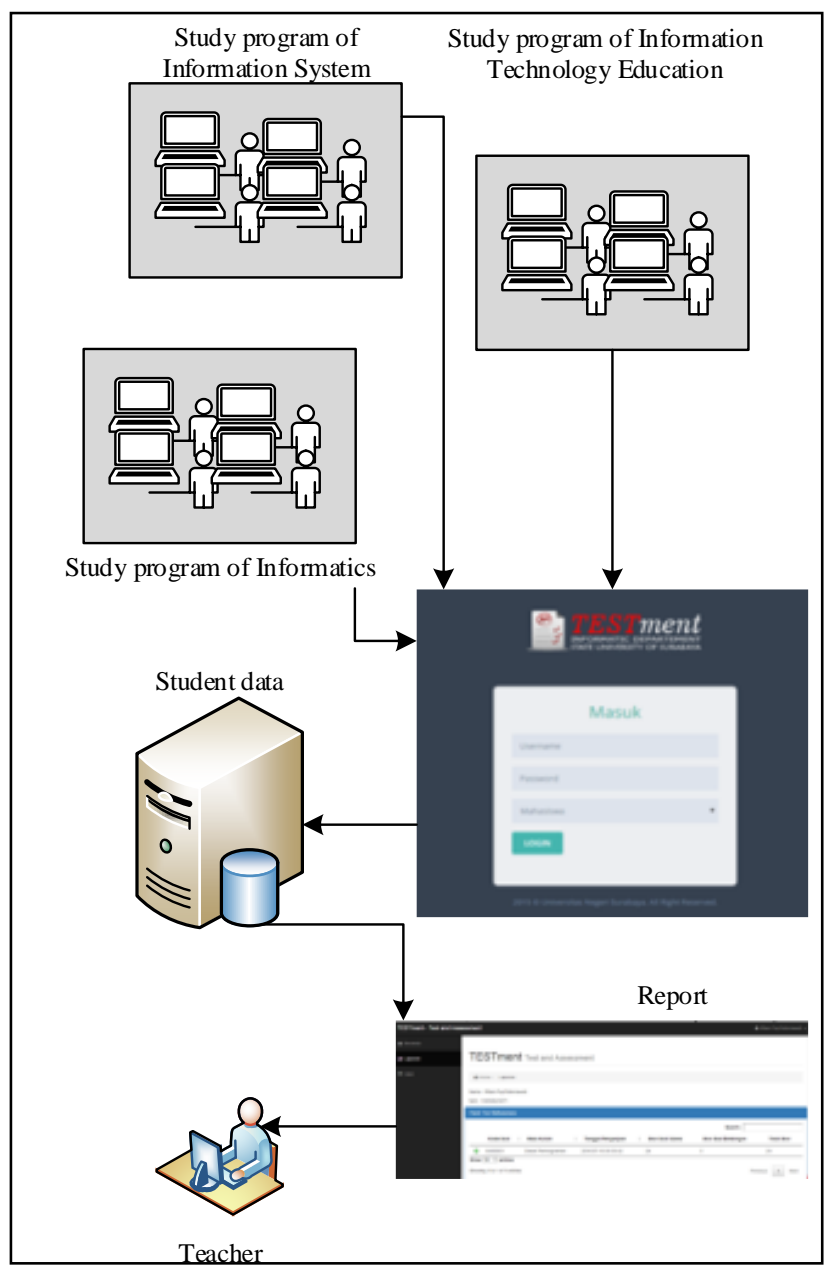

Figure. 2 The collection process of student data

In the second step, we combine the filter-based approach (FBSE) with wrapper-based an approach (WBSE) called HFS (Hybrid Feature Selection). Here, we want to reduce feature set $\mathrm{L}$ accumulated from both of them to produce features $\mathrm{O}$, where $\mathrm{O} \subseteq$ $\mathrm{N} \subseteq \mathrm{M}$.

For WBSE, classification algorithms or classifiers can be employed to build a wrapperbased approach (WBSE) to evaluate each set of features [38]. Therefore, in this research, we exploit classifiers, namely: one Rule (oneR) algorithm, to build this approach. This algorithm is simple and yet can achieve high accuracy [39]. one Rule evaluates the attribute worth method and then it ranks attributes by their evaluations. Besides, it does prediction using the minimum-error attribute.

\subsection{Clustering-based classification}

Generally, clustering is categorized as unsupervised learning, and it usually generates clusters evaluated by the unsupervised evaluation. Where the evaluation does not involve external information for example: label. In this case, especially on the clustering of the students' cognitive domain, we want to interpret clusters as classes. So, we can measure the performance of clustering by using classification metrics although our student data does not have a label (unlabeled data).

Therefore, we propose a clustering-based classification. Here, we adopt a simple metaclassifier that uses cluster algorithms as classifiers called clusterers. In this research, we apply cluster algorithms that use a fixed number of clusters, namely: K-means, EM, and Farthest-first. The code of a simple meta-classifier is based on the 'clusters to classes' functionality [40]. Generally, this method consists of 2 steps, namely: clustering for classification and measuring the resulted clusters using the classifier performance.

\subsection{Post-processing stage}

In this stage, we evaluate our proposed framework using two evaluation techniques, namely: the cross-validation and the percentage split. The evaluation results are measured by many metrics of classification, namely: Precision, Recall, F-Measure, MAE and Accuracy.

$$
\text { Precision }=\frac{T P}{T P+F P}
$$

$$
\begin{aligned}
& \text { Recall }=\frac{T P}{T P+F N} \\
& F-\text { Measure }=\frac{2 T P}{2 T P+F P+F N} \\
& \text { Accuracy }=\frac{T P+T N}{T P+T N+F P+F N}
\end{aligned}
$$

Where,

$\mathrm{TP}=$ True Positives is the number of positive predicted instances which are actually positive FP $=$ False Positives is the number of positive predicted instances which are actually negative $\mathrm{TN}=$ True Negatives is the number of negative predicted instances which are actually negative FN $=$ False Negatives is the number of negative predicted instances that are actually positive.

Then, we do the results visualization and analyze the misclassified student on all methods. Finally, we also record the time taken to build student modeling. So, we can analyze how long our 
proposed approach takes a faster time than the others.

\section{Result and discussion}

In this section, we describee the student data used for evaluation, the experimental result and metrics adopted to measure the performance of our proposed framework.

\subsection{Student data description}

Student data in this research are collected from the higher education in Surabaya, Indonesia, namely: Universitas Negeri Surabaya.

There are 98 students in the first semester of the Informatics department involved in this research. They come from 3 study programs, namely: Informatics, Information System and Technology Information Education. They interact with the evaluation system called the TESTment system. There are 10 the main questions and 30 the scaffolding questions (the guide questions to solve the main questions) entered by the teacher to the

Table 2. Features selection for Student data that its features extracted based on category

\begin{tabular}{|l|l|l|}
\hline \multicolumn{1}{|c|}{$\begin{array}{c}\text { Feature } \\
\text { Selection }\end{array}$} & $\begin{array}{c}\text { Sum of } \\
\text { Features }\end{array}$ & \multicolumn{1}{|c|}{ Feature Selection } \\
\hline $\begin{array}{l}\text { Original } \\
\text { Features }\end{array}$ & 10 & $\begin{array}{l}\text { MID, MID\%True, } \\
\text { Time_MID, } \\
\text { Score_MID, GID, } \\
\text { GID\%True, } \\
\text { Time_GID, Hint_GID, } \\
\text { Score_GID, } \\
\text { Score_Total }\end{array}$ \\
\hline $\begin{array}{l}\text { Features } \\
\text { Selection (FS) }\end{array}$ & 2 & MID, GID\%True \\
\hline $\begin{array}{l}\text { Hybrid Features } \\
\text { Selection (HFS) }\end{array}$ & 1 & MID \\
\hline
\end{tabular}

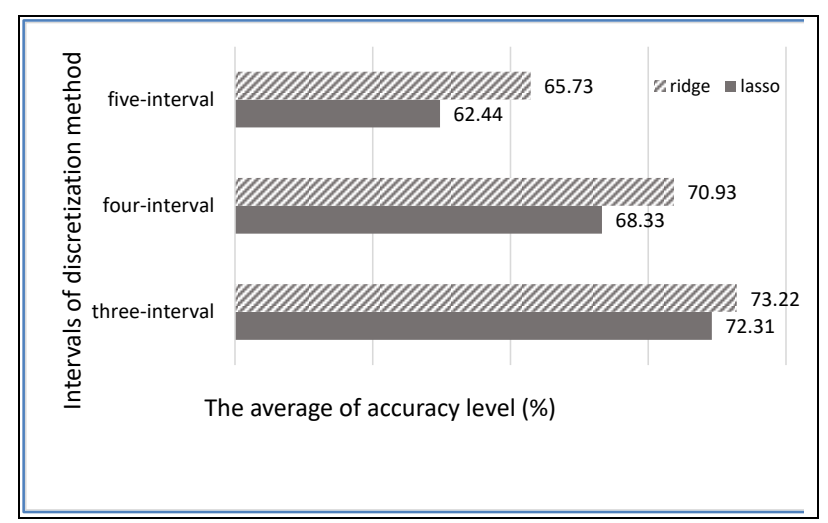

Figure. 5 Comparison of accuracy level average on all intervals using logistic regression system. Also, each main question contains three scaffolding questions. In this test, each scaffolding question provides hints which assist the student in solving the test item. All activities of the student are logged, and the processing time of each item is recorded. Therefore, there are 153 raw features stored in the database: MID1,.., MID10, MID\%True, Time MID1, ..., Time MID10, Score MID1,.., Score MID10, GID1.1,.., GID1.3,

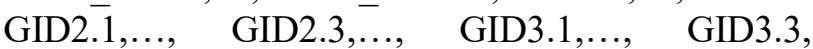
GID4.1,.., GID4.3, .., GID10.1,., GID10.3, GID\%True, Time_GID1.1,...Time_GID10.3,

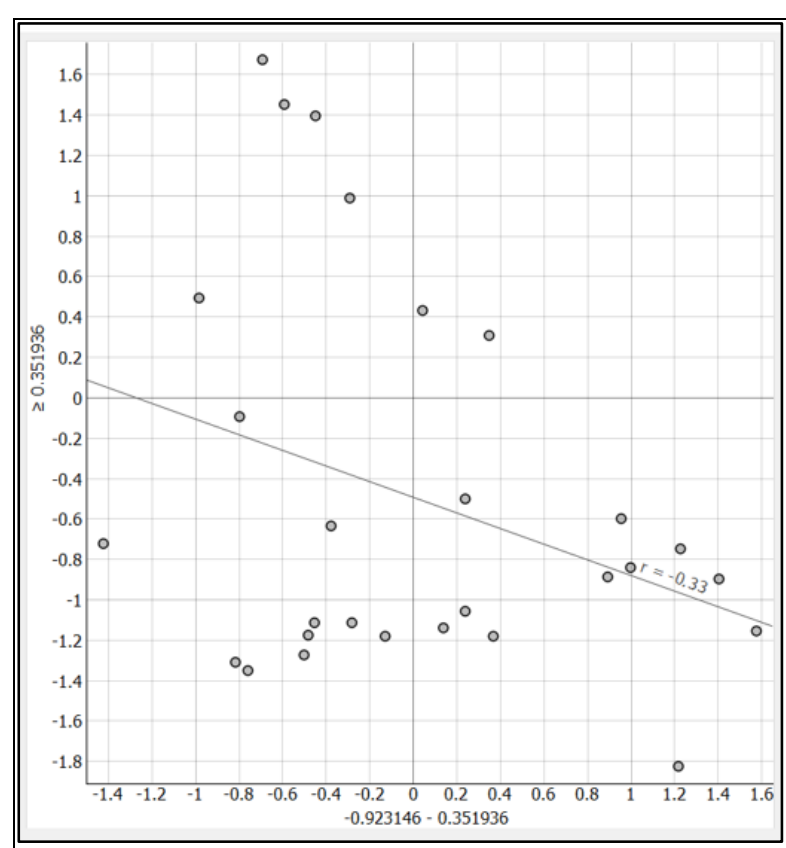

Figure. 3 Discretization-three interval-lasso

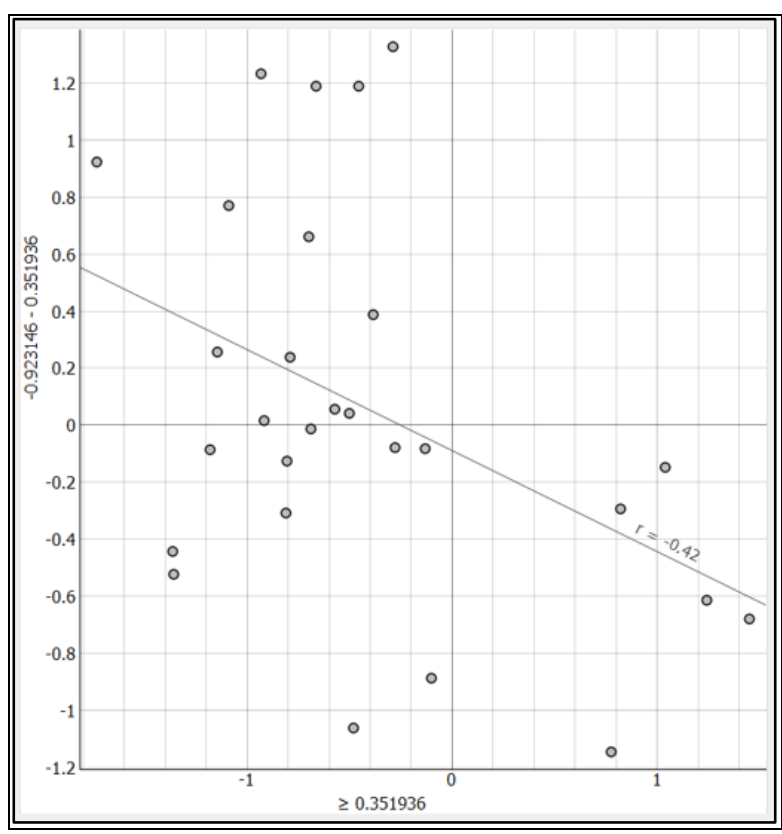

Figure. 4 Discretization-three interval-ridge 
Hint_GID1.1, ..., Hint_GID10.3, Score_GID1.1, ..., Score_GID10.3, Score_Total. These features are characteristics' student called as a student data.

Generally, student data is exploited to generate the students' achievement report. Further, our research explores the student data for building an effective model of students' cognitive domain. Generally, the process of data collecting is described in Fig. 2. Features of student data are extracted based on the category, as in the previous research [21] to improve the performance. This extraction is carried out by merging some features and calculating its average value with these formulas as follows, for $\mathrm{n}=$ the number of the main items, $\mathrm{m}=$ the number of scaffolding items.

$$
M I D=\frac{\sum_{i=1}^{n} M I D_{i}}{n}
$$

MID\%True $=\frac{\sum_{i=1}^{n}(\text { MID }=\text { True })}{n}$
Time_MID $=\frac{\sum_{i=1}^{n} \text { Time }_{-} M I D_{i}}{n}$

Score_MID $=\frac{\sum_{i=1}^{n} \text { Score }_{-} M I D_{i}}{n}$

$G I D=\frac{\sum_{i=1}^{n} \sum_{j=1}^{m} G I D_{i, j}}{n . m}$

GID\%True $=\frac{\sum_{i=1}^{n} \sum_{j=1}^{m}\left(G I D_{i, j}=\text { True }\right)}{n . m}$

Time_GID $=\frac{\sum_{i=1}^{n} \sum_{j=1}^{m} \text { Time_GID }_{i, j}}{n . m}$

Table 3. Performance of clustering-based classification on 4 metrics

\begin{tabular}{|l|l|l|l|l|l|l|l|l|}
\hline & \multicolumn{3}{|c|}{ Cross-validation technique (Average) } & \multicolumn{3}{c|}{ Percentage split technique (Average) } \\
\hline Method & Precision & Recall & F-Measure & MAE & Precision & Recall & F-Measure & MAE \\
\hline Ori_Kmeans & 0.5875 & 0.7143 & 0.6311 & 0.1911 & 0.5833 & 0.7305 & 0.638 & 0.1797 \\
\hline FS_Kmeans & 0.7079 & 0.692 & 0.6915 & 0.2054 & 0.6709 & 0.7962 & 0.7241 & 0.1394 \\
\hline HFS_Kmeans & $\mathbf{0 . 7 9 3 2}$ & $\mathbf{0 . 7 7 2 9}$ & $\mathbf{0 . 7 7 8 6}$ & $\mathbf{0 . 1 5 1 4}$ & $\mathbf{0 . 7 2 7 6}$ & $\mathbf{0 . 8 4 5 2}$ & $\mathbf{0 . 7 7 8 6}$ & $\mathbf{0 . 1 0 3 2}$ \\
\hline Ori_EM & 0.5959 & 0.7299 & 0.6449 & 0.1801 & 0.6003 & 0.7217 & 0.6057 & 0.1855 \\
\hline FS_EM & 0.8091 & 0.7775 & 0.785 & 0.1485 & 0.8086 & 0.8342 & 0.8118 & 0.1105 \\
\hline HFS_EM & $\mathbf{0 . 8 4 6 9}$ & $\mathbf{0 . 7 6 8}$ & $\mathbf{0 . 7 9 4 9}$ & $\mathbf{0 . 1 5 4 6}$ & $\mathbf{0 . 6}$ & $\mathbf{0 . 7 7 3 3}$ & $\mathbf{0 . 6 7 5 3}$ & $\mathbf{0 . 1 5 1 1}$ \\
\hline & & & & & & & & \\
\hline Ori_FarthestFirst & 0.5218 & 0.6561 & 0.6042 & 0.2587 & 0.5506 & 0.6593 & 0.5667 & 0.227 \\
\hline FS_FarthestFirst & 0.765 & 0.7813 & 0.762 & 0.1959 & 0.695 & 0.7221 & 0.67 & 0.1852 \\
\hline HFS_FarthestFirst & $\mathbf{0 . 7 9 3 3}$ & $\mathbf{0 . 7 9 1 1}$ & $\mathbf{0 . 7 7 7 9}$ & $\mathbf{0 . 1 3 9 2}$ & $\mathbf{0 . 7 5 4 9}$ & $\mathbf{0 . 7 8 5 6}$ & $\mathbf{0 . 7 3 8 3}$ & $\mathbf{0 . 1 4 3}$ \\
\hline
\end{tabular}

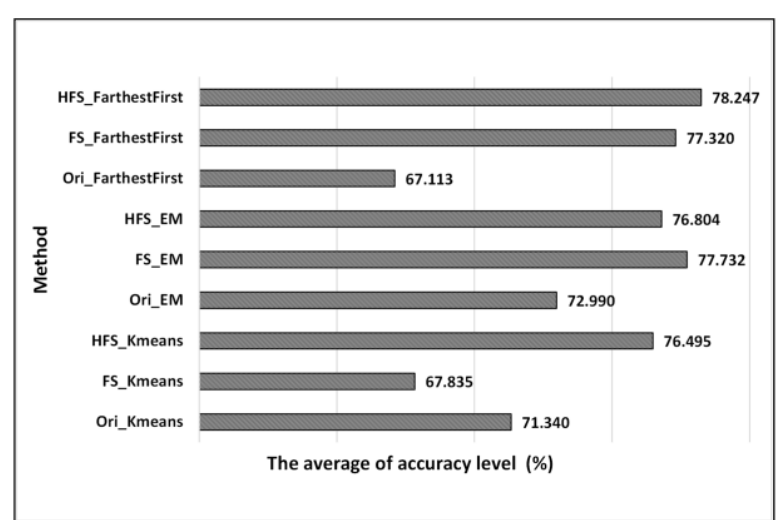

Figure. 6 Comparison of accuracy level average on cross-validation technique

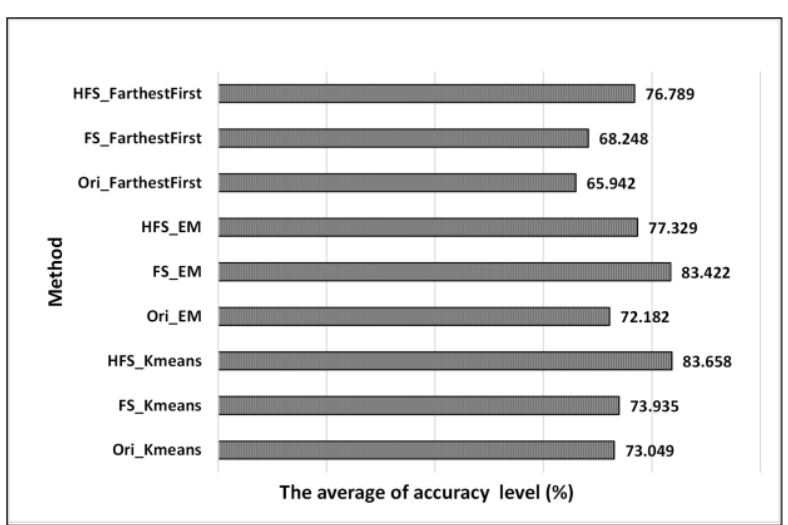

Figure. 7 Comparison of accuracy level average on percentage split technique 


$$
\begin{gathered}
\text { Hint_GID }=\frac{\sum_{i=1}^{n} \sum_{j=1}^{m} \text { Hint_GID } D_{i, j}}{n . m} \\
\text { Score_GID }=\frac{\sum_{i=1}^{n} \sum_{j=1}^{m} \text { Score_GID }{ }_{i, j}}{n . m}
\end{gathered}
$$

Score_Total $=M I D+G I D$

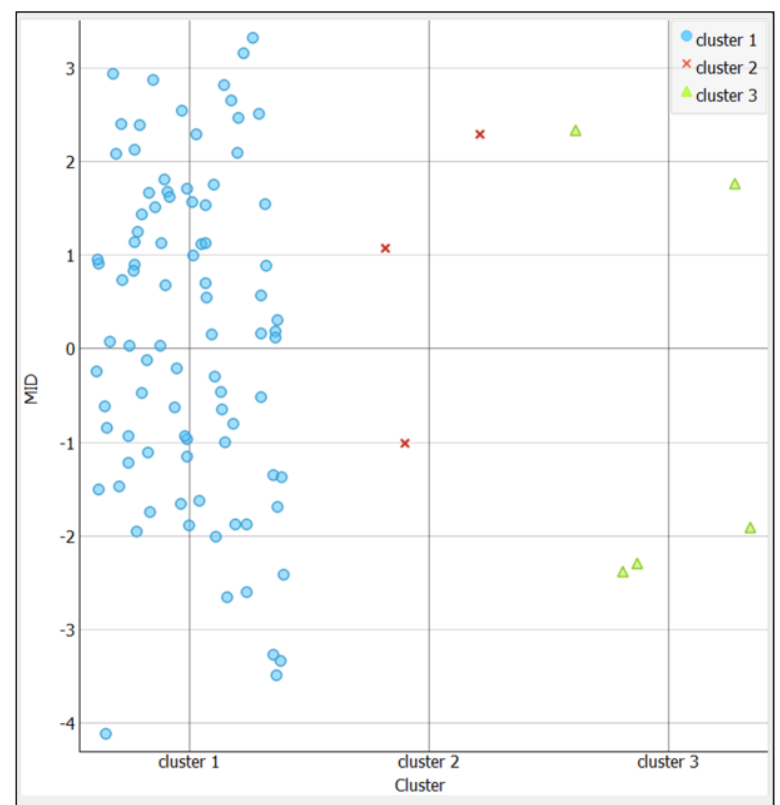

Figure. 8 The best mapping result on cross-validation technique (HFS features and Farthest first on fold 10)

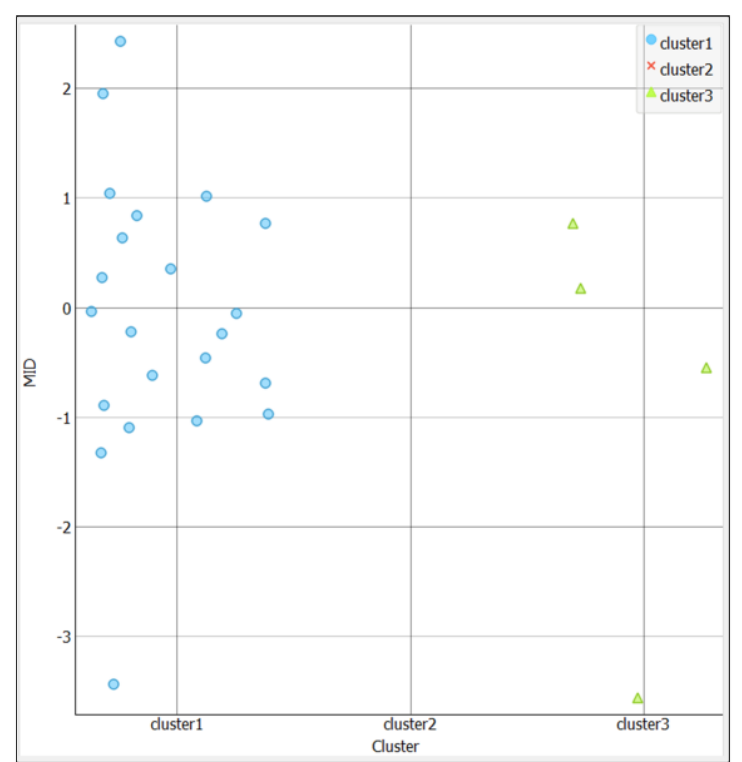

Figure. 10 The best mapping result on percentage split technique (HFS feature and K-means on split 73)
From this step, we obtain 10 new features as Original Features (see Table 2.) which represent the raw 153 features. This method has significantly reduced the number of execution time and enhanced the accuracy level.

\subsection{Identifying the dominant characteristics}

In the pre-processing phase, there are two methods proposed. They are the discretization method on the logistic regression and the combination of features selection methods.

Firstly, we apply the discretization method on the logistic regression to support in labeling step.

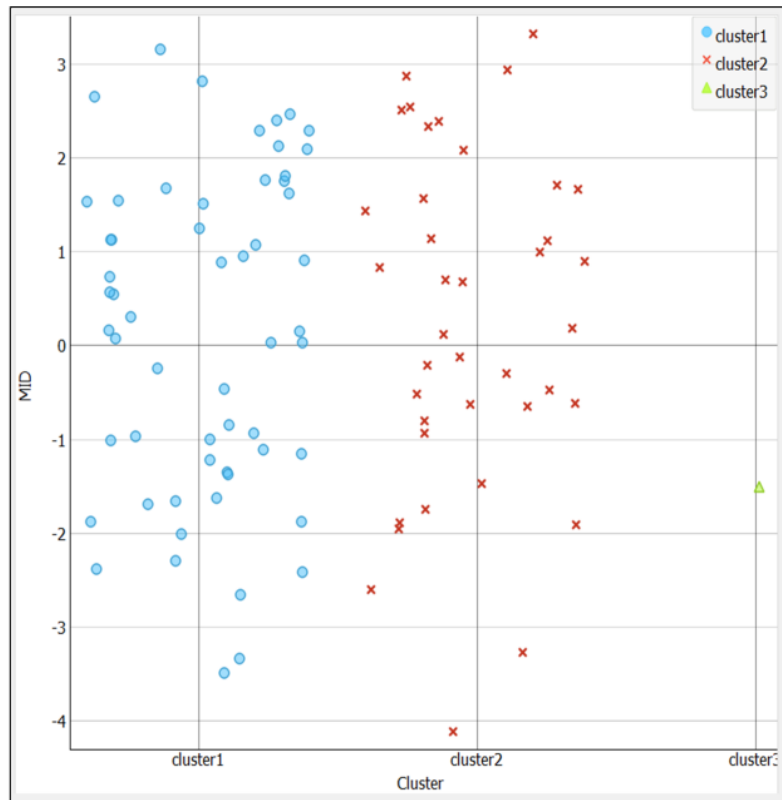

Figure. 9 The worst mapping result on cross-validation technique (Original features and Farthest first on fold 6)

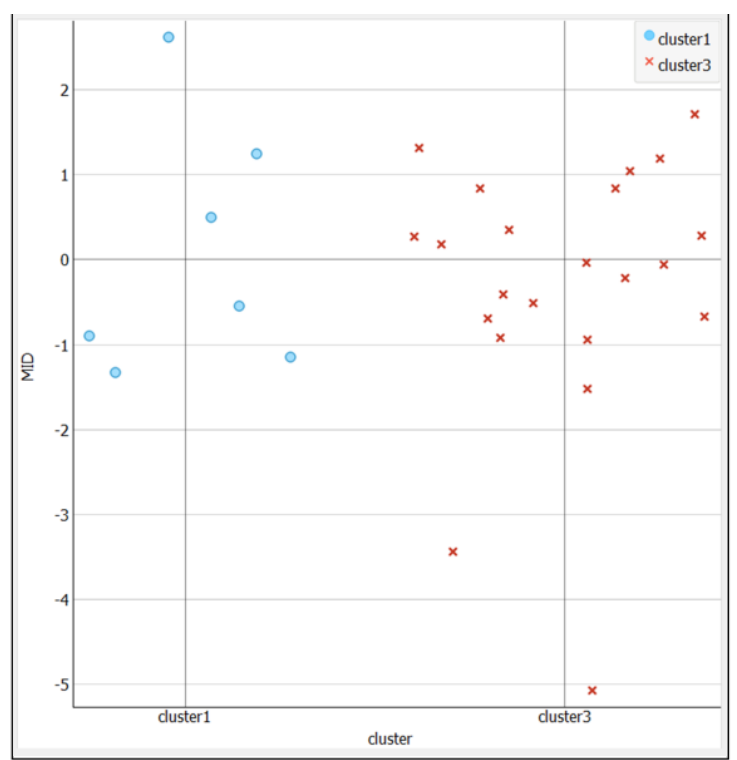

Figure. 11 The worst mapping result on percentage split technique (FS features and Farthest first on split 70) 
Furthermore, this method is explored to decide the number of clusters to obtain the optimal clustering process. Here, we adopt the discretization method which is called equal-width to discretize the continuous data on student data extracted based on category. Additionally, we do the discretization for three-interval, four-interval, and five-interval as extended to the previous work [26]. For evaluating, we build the logistic regression with two regularizations: lasso, ridge.

Additionally, we explore percentage split as the evaluation technique with training size: $10 \%, 20 \%$, $30 \%, 40 \%, 50 \%$ and $60 \%$ on train/test repeating: 2 and 3. Metrics adopted for evaluation of the discretization method is the correlation and average accuracy level. For correlation metric (r-value), three-interval combined by logistic regression achieves the highest value on both regularization lasso and ridge.

Here, the $\mathrm{XY}$-axis is divided into three intervals, namely: $\geq 0.351936,-0.923146-0.351936$, <0.923146 . The experimental result shows that lasso and ridge are $r=-0.33$ and $r=-0.42$, respectively, described in Figs. 3-4. Four-interval on lasso and ridge are $r=-0.22$ and $r=-0.42$. Lastly, on fiveinterval, lasso and ridge are $r=0.25$ and $r=0.19$, respectively.

Further, we compare the average accuracy level on all intervals and regularizations as shown in Fig. 5. The experimental result indicates that threeinterval achieves the highest value for all regularizations, namely: ridge $=72.31 \%$ and lasso $=$ $73.22 \%$. This means that the most optimal number cluster is three clusters.

Secondly, we exploit features selection methods to find relevant features which are dominant characteristics of the students' cognitive domain. Here, we propose Hybrid Features Selection (HFS) which is a combination of both the filter-based and wrapper-based approach. The result of this step is depicted in Table 2. It is found that FS and HFS eliminate 8 and 9 irrelevant features of 10 original features.

\subsection{Evaluating the clustering-based classification performance}

To evaluate what these features are dominant characteristics of a students' cognitive domain, we propose the clustering-based classification model to measure the result with the more metrics. Besides, our proposed approach can generate information relating to the sum of misclassified students. Here, we employ two evaluation techniques, namely: cross-validation and percentage split. We do the cross-validation on fold 3-12 and the percentage split on split 66\%, 70\%, 73\%, 75\%, 78\%, 80\%, 83\%, $85 \%, 88 \%$ and $90 \%$. The evaluation results on both techniques are presented on four metrics, namely: precision, recall, F-measure and MAE, described in Table 3. HFS feature achieves the highest level on almost three metrics, namely: precision, recall and F-Measure. Additionally, the lowest level of MAE metric illustrating the prediction error level also is reached by the HFS feature. It means that the HFS feature is a dominant characteristic.

Additionally, we also measure the performance by the accuracy level. In general, the accuracy level of both techniques fluctuates. In detail, it is found that the highest accuracy level on the crossvalidation technique is achieved by the combination of HFS features and Farthest first on fold 10 about $86.5979 \%$. On the contrary, the lowest accuracy level is reached by the combination of original features and farthest first on fold 6 about $55.6701 \%$.

In another technique, the highest accuracy level is reached by the combination of HFS features and K-means about 96.1538\%. Inversely, the lowest accuracy level is achieved by the combination of FS features and Farthest first about $37.931 \%$. It is found that the HFS feature is the dominant characteristic of students' cognitive indicated by the HFS feature achieves the highest accuracy level on all evaluation techniques.

In addition, we also count the accuracy level average on all folds and all splits for every method. The experiment results describe that the HFS feature outperforms higher value than the others on two clustering methods of three clustering methods applied on both evaluation techniques as shown in Figs. 6 and 7. On the cross-validation technique depicted in Fig. 6, the HFS feature achieves the highest accuracy level average compared by the others when it is combined by Farthest first and Kmeans about $78.247 \%$ and $76.495 \%$, respectively. Especially, on the EM method, the HFS feature achieves a lower accuracy level average than FS features. On the contrary, original features have the lowest position in terms of the accuracy level average on Farthest first and EM about $67.113 \%$ and $72.990 \%$.

So, the HFS feature can improve the model performance of students' cognitive domain optimally, in terms of the accuracy level average, when it is combined by Farthest first and K-means. On Kmeans, the HFS feature can improve the higher accuracy level average than original features and FS features about $5.1546 \%$ and $8.6598 \%$, respectively. While, on Farthest first, the HFS feature can improve the higher accuracy level average than FS 
features and original features around $0.92785 \%$ and $11.13402 \%$.

The percentage split describes the same result with the cross-validation. The HFS feature also reaches the highest of accuracy level average when this feature is combined by Farthest first and Kmeans, namely: about $78.247 \%$ and $76.495 \%$, respectively. On the contrary, the HFS feature only achieves a lower accuracy level average than FS features on the EM method. Conversely, the lowest accuracy level average is achieved by original features on all clustering methods, namely: Farthest first, EM and K-means about $65.942 \%, 72.182 \%$, and $73.049 \%$, respectively.

This means that the HFS feature can improve the model performance of students' cognitive domain significantly, in terms of the accuracy level average, when this feature is combined by Farthest first and K-means. Also, on K-means, the HFS feature can improve the performance, in terms of accuracy level average than original features and FS features about $10.609 \%$ and 9.724\%, respectively. The combination of HFS feature and Farthest first can improve the higher accuracy level average than FS features and original features around $8.541 \%$ and $10.847 \%$. Inversely, the performance of students' cognitive domain reaches the lower of accuracy level average than the HFS feature when original features are combined by all clustering methods, namely: Farthest first about $10.847 \%$, EM about $5.148 \%$ and K-means $10.609 \%$. This indicates that the HFS feature is the dominant characteristic of students' cognitive domain.

Furthermore, we visualize the best result and the worst result of a students' cognitive domain clustering based on the accuracy level depicted in Figs. 8-11. We also analyze the sum of misclassified

Table 4. Comparison of the sum average of inappropriate students' cognitive domain

\begin{tabular}{|c|c|c|}
\hline \multirow[b]{2}{*}{ Method } & \multicolumn{2}{|c|}{$\begin{array}{l}\text { The sum average of the } \\
\text { misclassified students }\end{array}$} \\
\hline & $\begin{array}{l}\text { Cross- } \\
\text { validation }\end{array}$ & $\begin{array}{c}\text { Percentage } \\
\text { split }\end{array}$ \\
\hline Ori_Kmeans & 28 & 6 \\
\hline FS_Kmeans & 29 & 3 \\
\hline HFS_Kmeans & 22 & 3 \\
\hline Ori_EM & 26 & 6 \\
\hline FS_EM & 22 & 4 \\
\hline HFS_EM & 23 & 5 \\
\hline Ori_FarthestFirst & 32 & 8 \\
\hline FS_FarthestFirst & 21 & 6 \\
\hline HFS_FarthestFirst & 20 & 5 \\
\hline
\end{tabular}

students. The visualization is done on the crossvalidation technique and the percentage split depicted in Figs. 8 and 9 and on Figs. 10 and 11, respectively.

The best mapping of students' cognitive domain on the cross-validation is generated by the combination of the HFS feature and the Farthest first on fold 10 described in Fig. 8. The clusters' composition is as follows; cluster 1: 89 students, cluster 2: 3 students, cluster 3: 5 students. The sum of the misclassified student is 13 students. On the contrary, the worst mapping of students' cognitive domain on the cross-validation is obtained by the combination of the original features and the Farthest first on fold 6 illustrated in Fig. 9. The composition of cluster 1, cluster 2, and cluster 3 are 57, 39 and 1 student, respectively. In addition, this model produces the sum of the misclassified student, namely: 43 students.

On the percentage split, the best mapping of students' cognitive domain is generated by the combination of the HFS feature and K-means on split 73 illustrated in Fig. 10. The composition of all clusters is as follows; cluster 1: 22 students, cluster 2: 0 students, cluster 3: 4 students. Additionally, the sum of the misclassified student is only 1 student.

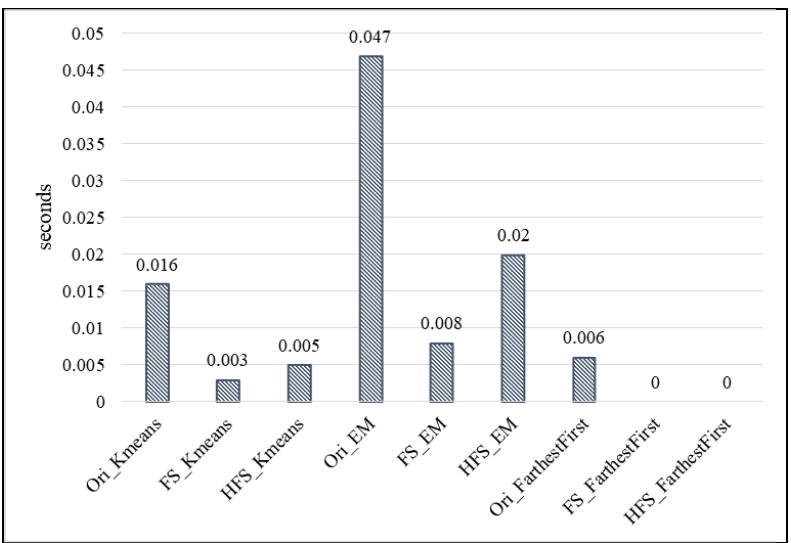

Figure. 12 Comparison of time taken to build a model on cross-validation technique

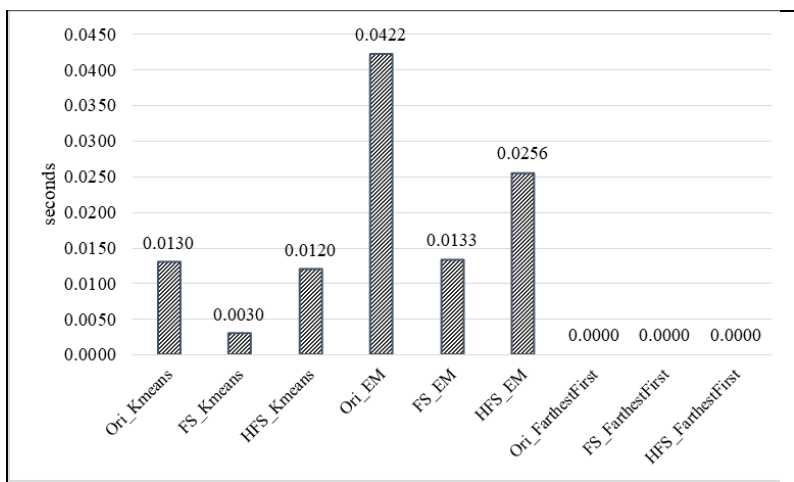

Figure. 13 Comparison of time taken to build a model on percentage split technique 
On the contrary, the worst mapping of students' cognitive domain is obtained by the combination of the FS features and Farthest first on the percentage split 70 depicted in Fig. 11. The composition of cluster 1 , cluster 2 , and cluster 3 are 7, 0 and 20 students, respectively. In addition, this model produces the sum of the misclassified students, namely: 18 students. Here, the fewest sums of misclassified students on both evaluation techniques are generated by the HFS feature.

Overall, the HFS feature has the lowest sum average of misclassified students as shown in Table 4. Here, we can infer that the HFS feature can decrease the misclassified students significantly on $\mathrm{K}$-means and Farthest first. On the cross-validation, the reduction sums of the misclassified student are 6 students from 28 to 22 for the combination of HFS and K-means. Next, the combination of HFS and Farthest first can decrease 12 misclassified students from 32 to 20 . On the split percentage, the reduction sums of the misclassified student are 3 students from 6 to 3 on K-means and form 8 to 5 on Farthest first. This indicates that the HFS feature is the dominant characteristic because this feature can improve the mapping of students' cognitive domain.

Finally, we also record the time needed to build the student modeling for all methods on both evaluation techniques as depicted in Figs. 12 and 13. Here, almost all the combination of FS features and clustering methods achieve the lowest time taken to build the model on both evaluation techniques. On the contrary, the highest time taken is reached by the combination of original features and all clustering methods on all evaluation techniques. Furthermore, the lowest time taken is achieved by FS features combined by clustering methods: K-means about 0.003 seconds and EM about 0.008 seconds using the cross-validation technique described in Fig. 12. The same results also occur on the FS features exploited using the percentage split. FS features reach the lowest time taken to build the model when they are combined by K-means around 0.003 seconds and EM around 0.0133 seconds depicted in Fig. 13. Inversely, original features combined by all clustering methods achieve the highest time taken on all evaluation techniques as shown in Figs. 12 and 13. Here, the longest duration occurs on the model built by the combination of original features and EM for both evaluation techniques, namely: on the cross-validation around 0.047 seconds and the percentage split around 0.0422 seconds.

In addition, the HFS only achieves the lowest time taken to build the model on Farthest first using the cross-validation and percentage split about 0 seconds. This happened because the time taken needed in milliseconds, while our system records in seconds form. On the other methods, FS features still achieve the lower time taken than the HFS feature. This means that FS features can reduce optimally the time taken on all combinations. However, in the experimental results, the HFS feature combined by all methods reaches the lower time taken than the original features combined by all methods in all cases. This is indicated by the HFS feature on cross-validation that can reduce the time taken to build the model on Farthest first about 0.006 seconds, on EM about 0.027 seconds and on K-means about 0.011 seconds. Additionally, on the percentage split, the HFS feature also decreases the time taken on EM around 0.0167 and $\mathrm{K}$-means around 0.0010 seconds.

This indicates that the HFS feature is faster than Original features for building the student modeling relating to mapping the students' cognitive domain.

From the aforementioned results, we conclude that our proposed approach provides excellent identification of dominant characteristics indicated by the optimal value on all metrics of the classifiers.

\section{Conclusions}

This research demonstrates that our proposed approach successfully identifies relevant features as dominant characteristics. In terms of classifier metrics, our method can improve the performance of clustering-based classification significantly. On the cross-validation for Farthest first on both the evaluation techniques, our method is about 11.134 percent higher in terms of accuracy average level than the original features. Then, our method can also enhance this metric about 10.847 percent than the original features on the percentage split. So, this model generated can decrease the sum of inappropriate students based on the cognitive domain significantly. On the cross-validation, the reduction sums of the misclassified student are 6 from 28 to 22 students for the combination of HFS and K-means. Next, the combination of HFS and Farthest first can decrease by about 12 from 32 to 20 students. On the split percentage, the reduction sums of the misclassified student are 3 students from 6 to 3 on K-means and from 8 to 5 on Farthest first.

Furthermore, this method reduces drastically the time taken to build a model based on the crossvalidation and percentage split when HFS is combined on EM around 0.027 and 0.0167 seconds, respectively. Finally, the teacher can map this domain simpler and faster than that of using all features. 


\section{Acknowledgments}

The authors would like to thank Indonesia Endowment Fund BUDI-DN/LPDP for Education for their support through the Indonesian education scholarship program, and also the Ministry of Research, Technology, and Higher Education through the scholarship of Enhancing International Publication Program (PKPI / sandwich-like) 2018.

\section{References}

[1] M. W. Rodrigues, L. E. Zárate, and S. Isotani, "Educational Data Mining: A review of evaluation process in the e-learning", Telemat. Informatics, 2018.

[2] A. Peña-Ayala, Educational data mining: Applications and trends. 2014.

[3] P. Dillenbourg, "The Evolution of Research on Digital Education", Int. J. Artif. Intell. Educ., Vol. 26, No. 2, pp. 544-560, 2016.

[4] R. Cerezo, M. Sánchez-Santillán, M. P. PauleRuiz, and J. C. Núñez, "Students' LMS interaction patterns and their relationship with achievement: A case study in higher education", Comput. Educ., Vol. 96, pp. 42-54, 2016.

[5] S. Natek and M. Zwilling, "Student data mining solution-knowledge management system related to higher education institutions", Expert Syst. Appl., Vol. 41, No. 14, pp. 6400-6407, 2014.

[6] C. Troussas, M. Virvou, and S. Mesaretzidis, "Comparative Analysis of algorithms for student characteristics classification using a Methodological Framework", In: Proc. of the 6th International Conference on Information, Intelligence, Systems and Applications (IISA), 2015.

[7] R. Harimurti, Y. Yamasari, Ekohariadi, Munoto, and B. I. G. P. Asto, "Predicting student's psychomotor domain on the vocational senior high school using linear regression", In: Proc. of 2018 International Conference on Information and Communications Technology (ICOIACT), pp. 448-453, 2018.

[8] F. Ahmad, N. H. Ismail, and A. A. Aziz, "The prediction of students' academic performance using classification data mining techniques", Appl. Math. Sci., Vol. 9, pp. 6415-6426, 2015.

[9] B. Guo, R. Zhang, G. Xu, C. Shi, and L. Yang, "Predicting students performance in educational data mining", In: Proc. of International Symposium on Educational Technology, ISET 2015, 2016.

[10] M. Wati, W. Indrawan, J. A. Widians, and N. Puspitasari, "Data mining for predicting students' learning result", In: Proc. of 2017 4th International Conference on Computer Applications and Information Processing Technology (CAIPT), 2017, pp. 1-4.

[11] E. B. Costa, B. Fonseca, M. A. Santana, F. F. de Araújo, and J. Rego, "Evaluating the effectiveness of educational data mining techniques for early prediction of students' academic failure in introductory programming courses", Comput. Human Behav., Vol. 73, pp. 247-256, 2017.

[12] Y. Promdee, S. Kasemvilas, N. Phangsuk, and R. Yodthasarn, "Predicting Persuasive Message for Changing Student's Attitude Using Data Mining", In: Proc. of 2017 International Conference on Platform Technology and Service (PlatCon), pp. 1-5, 2017.

[13] W. Xing, R. Guo, E. Petakovic, and S. Goggins, "Participation-based student final performance prediction model through interpretable Genetic Programming: Integrating learning analytics, educational data mining and theory-ARM", Comput. Human Behav., Vol. 47, pp. 168-181, 2015.

[14] R. Asif, A. Merceron, S. A. Ali, and N. G. Haider, "Analyzing undergraduate students' performance using educational data mining", Comput. Educ., Vol. 113, pp. 177-194, 2017.

[15] N. T. Pang, M. Steinbach, and V. Kumar, Introduction to Data mining. 2006.

[16] J. Han, M. Kamber, and J. Pei, Data Mining Concepts and Techniques. USA: Elsevier, 2012.

[17] I. Singh, A. S. Sabitha, and A. Bansal, "Student performance analysis using clustering algorithm", In: Proc. of 2016 6th International Conference - Cloud System and Big Data Engineering (Confluence), pp. 294-299, 2016.

[18] L. Najdi and B. Er-Raha, "Implementing cluster analysis tool for the identification of students typologies", In: Proc. of 2016 4th IEEE International Colloquium on Information Science and Technology (CiSt), pp. 575-580, 2016.

[19] Z. Li, C. Shang, and Q. Shen, "Fuzzy-clustering embedded regression for predicting student academic performance", In: Proc. of 2016 IEEE International Conference on Fuzzy Systems (FUZZ-IEEE), pp. 344-351, 2016.

[20] Z. Fan and Y. Sun, "Clustering of College Students Based on Improved K-Means Algorithm", In: Proc. of 2016 International Computer Symposium (ICS), pp. 676-679, 2016.

[21] Y. Yamasari, S. M. S. Nugroho, I. N. Sukajaya, and M. H. Purnomo, "Features extraction to improve performance of clustering process on 
student achievement", In: Proc. of 2016 International Computer Science and Engineering Conference (ICSEC), pp. 1-5, 2016.

[22] M. Singh, J. Singh, and A. Rawal, "Feature Extraction Model to Identify At -- Risk Level of Students in Academia", In: Proc. of 2014 International Conference on Information Technology, pp. 221-227, 2014.

[23] Y. Yamasari, S. M. S. Nugroho, R. Harimurti, and M. H. Purnomo, "Improving the cluster validity on student's psychomotor domain using feature selection", In: Proc. of 2018 International Conference on Information and Communications Technology (ICOIACT), pp. 460-465, 2018.

[24] M. Zaffar, M. A. Hashmani, and K. S. Savita, "Performance analysis of feature selection algorithm for educational data mining", In: Proc. of 2017 IEEE Conference on Big Data and Analytics (ICBDA), pp. 7-12, 2017.

[25] A. Pradeep, S. Das, and J. J. Kizhekkethottam, "Students dropout factor prediction using EDM techniques", In: Proc. of the IEEE International Conference on Soft-Computing and Network Security, ICSNS 2015, 2015.

[26] Y. Yamasari, P. W. Rusimamto, N. Rochmawati, D. F. Suyatno, S. C. Wibawa, S. M. S. Nugroho, and M.H. Purnomo, "Discretization method to optimize logistic regression on classification of student's cognitive domain", MATEC Web Conf., Vol. 197, p. 3006, 2018.

[27] W. Punlumjeak and N. Rachburee, "A comparative study of feature selection techniques for classify student performance", In: Proc. of 2015 7th International Conference on Information Technology and Electrical Engineering (ICITEE), pp. 425-429, 2015.

[28] C. Pong-Inwong and K. Kaewmak, "Improved sentiment analysis for teaching evaluation using feature selection and voting ensemble learning integration", In: Proc. of 2016 2nd IEEE International Conference on Computer and Communications (ICCC), pp. 1222-1225, 2016.

[29] L. Rahman, N. A. Setiawan, and A. E. Permanasari, "Feature selection methods in improving accuracy of classifying students' academic performance", In: Proc. of 2017 2nd International conferences on Information Technology, Information Systems and Electrical Engineering (ICITISEE), pp. 267271, 2017.

[30] K. Deepika and N. Sathyanarayana, "Relief-F and Budget Tree Random Forest Based Feature
Selection for Student Academic Performance Prediction", International Journal of Intelligent Engineering and Systems, Vol. 12, No. 1, pp. 30-39, 2019.

[31] S. Shankar, B. D. Sarkar, S. Sabitha, and D. Mehrotra, "Performance analysis of student learning metric using K-mean clustering approach K-mean cluster", In: Proc. of 2016 6th International Conference - Cloud System and Big Data Engineering (Confluence), pp. 341-345, 2016.

[32] I. N. Sukajaya, I. K. E. Purnama, and M. H. Purnomo, "Intelligent classification of learner's cognitive domain using bayes net, naive bayes, and j48 utilizing bloom's taxonomy-based serious game", Int. J. Emerg. Technol. Learn., Vol. 10, No. 2, pp. 46-52, 2015.

[33] P. H. P. Rosa and R. Gunawan, "The Clustering of High Schools Based on National and School Examinations", In: Proc. of International Conference on Data and Software Engineering, pp. 231-236, 2015.

[34] R. Campagni, D. Merlini, R. Sprugnoli, and M. C. Verri, "Data mining models for student careers", Expert Syst. Appl., Vol. 42, No. 13, pp. 5508-5521, 2015.

[35] H. Harwati, A. P. Alfiani, and F. A. Wulandari, "Mapping Student $\hat{a} €^{\mathrm{TM}}$ s Performance Based on Data Mining Approach ( A Case Study)", Procedia - Social and Behavioral Sciences, Vol. 38, No. 1, pp. 173-177, 2015.

[36] Y. Park, J. H. Yu, and I.-H. Jo, "Clustering blended learning courses by online behavior data: A case study in a Korean higher education institute", Internet High. Educ., Vol. 29, pp. 1$11,2016$.

[37] M. A. Hall, "Correlation-based feature selection for machine learning", Ph.D. Thesis, The University of Waikato, 1999.

[38] M. H. Kamarudin, C. Maple, T. Watson, and N. S. Safa, "A LogitBoost-Based Algorithm for Detecting Known and Unknown Web Attacks", IEEE Access, Vol. 5, pp. 26190-26200, 2017.

[39] R. C. Holte, "Very Simple Classification Rules Perform Well on Most Commonly Used Datasets", Machine Learning, Vol. 11, pp. 6390, 1993

[40] I. H. Witten, E. Frank, and M. a. Hall, Data Mining: Practical Machine Learning Tools and Techniques, Third Edition, Vol. 54, No. 2. 2011.

[41] H. Park, S. Lee, M. Lee, M. Chang, and H. Kwak, "Computers in Human Behavior Using eye movement data to infer human behavioral intentions", Comput. Human Behav., Vol. 63, pp. 796-804, 2016. 